\title{
Tensor force effects and high-momentum components in the nuclear symmetry energy
}

\author{
A. Carbone ${ }^{1}$, A. Polls ${ }^{1}$, C. Providência ${ }^{2}$, A. Rios ${ }^{3}$, and I. Vidaña ${ }^{2}$ \\ 1 Department d'Estructura i Constituents de la Matèria and Institut de Ciències del Cosmos, Facultat de Física, Universitat \\ de Barcelona, E-08028 Barcelona, Spain \\ 2 Centro de Física Computacional, Department of Physics, University of Coimbra, PT-3004-516 Coimbra, Portugal \\ 3 Department of Physics, Faculty of Engineering and Physical Sciences, University of Surrey, Guilford GU2 7XH, United \\ Kingdom
}

Received: date / Revised version: date

\begin{abstract}
We analyze microscopic many-body calculations of the nuclear symmetry energy and its density dependence. The calculations are performed in the framework of the Brueckner-Hartree-Fock and the SelfConsistent Green's Functions methods. Within Brueckner-Hartree-Fock, the Hellmann-Feynman theorem gives access to the kinetic energy contribution as well as the contributions of the different components of the nucleon-nucleon interaction. The tensor component gives the largest contribution to the symmetry energy. The decomposition of the symmetry energy in a kinetic part and a potential energy part provides physical insight on the correlated nature of the system, indicating that neutron matter is less correlated than symmetric nuclear matter. Within the Self-Consistent Green's Function approach, we compute the momentum distributions and we identify the effects of the high momentum components in the symmetry energy. The results are obtained for the realistic interaction Argonne V18 potential, supplemented by the Urbana IX three-body force in the Brueckner-Hartree-Fock calculations.
\end{abstract}

PACS. 21.30.Fe; 21.65.Cd; 21.65.Ef; 21.65.Mn

Symmetry Energy, Tensor force, High-momentum components.

\section{Introduction}

The nuclear symmetry energy, defined as the difference between the energy per particle of pure neutron matter (PNM) and symmetric nuclear matter (SNM), and, in particular, its density dependence, is a crucial ingredient to understand many properties of isospin-rich nuclei and neutron stars 1,2. A major scientific experimental and theoretical effort is being devoted to study the properties of asymmetric nuclear systems. Laboratory experiments, such as those recently performed or being planned in existing or next-generation radioactive ion beam facilities such as the Facility for Antiproton and Ion Research (FAIR, Germany), Rikagaku Kenkyusho (RIKEN, Japan), the Heavy Ion Research Facility in Lanzhou (HIRFL, China), SPIRAL2 at the Grand Accelerateur National d'Ions Lourds (GANIL, France), and the upcoming Facility for Rare Isotope Beams (FRIB, Michigan State University) can probe the density behavior of the symmetry energy [1. More precisely, experimental information on the density dependence of the symmetry energy below, close to and above the saturation density of nuclear matter can be obtained from isospin diffusion measurements 3 , giant 4] and pigmy resonances [5], isobaric analog states [6], isoscaling [7] or meson production in heavy ion colli- sions 8, 9. Moreover, the accurate measurements of the neutron skin thickness in ${ }^{208} \mathrm{~Pb}$ via parity-violating electron scattering (PREX experiment) [10,11] or by means of antiprotonic atom data [12,13, also constrain the density dependence of the symmetry energy because of the socalled Typel-Brown correlation [14. A recent update and a critical analysis of these constraints on the nuclear symmetry energy can be found in Ref. 15]. Further details on these and other methods are given in other contributions to this special volume.

Additional information on the symmetry energy can be gathered from astrophysical observations of compact objects, which open a new window into both the bulk and microscopic properties of nuclear matter at extreme isospin asymmetries [2. In fact, the symmetry energy determines to a large extent the composition of $\beta$-stable matter and therefore the structure and mass of neutron stars [16. In particular, the characterization of the core-crust transition in neutron stars [17, 18, 19,20, or the analysis of power-law correlations, such as the relation between the radius of a neutron star and the equation of state [21], can put stringent constraints on the symmetry energy. From the theoretical point of view, the symmetry energy has been determined using both phenomenological and microscopic many-body approaches. Phenomenological ap- 
proaches, either relativistic or non-relativistic, are based on effective interactions that are usually fit to reproduce the binding energy of stable nuclei [22. Therefore, predictions at high asymmetries should be taken with care. The Skyrme-Hartree-Fock [23] and the Relativistic Mean Field 24 methods are the most common ones. However, in spite of the large amount of constraints imposed in the fitting procedures of the effective interactions, there is still a large dispersion on the results for the symmetry energy (and its derivatives) provided by the phenomenological approaches. Hence, fully microscopic approaches look as a safe and necessary alternative.

Microscopic approaches start from realistic nucleonnucleon $(\mathrm{NN})$ interactions that reproduce the scattering and bound state properties of the free two-nucleon system. In-medium correlations are then built using manybody techniques that incorporate the effects of the nuclear medium and account for isospin asymmetry effects such as, for instance, the difference in the Pauli blocking factors of neutrons and protons in asymmetric matter 25. Among this type of approaches the most popular ones are the Brueckner-Bethe-Goldstone (BBG) 26] and the Dirac-Brueckner-Hartree-Fock 27] theories, the variational method [28, the correlated basis function formalism 29], the self-consistent Green's function technique (SCGF) 30. or, recently, perturbative calculations using $\mathrm{V}_{\text {lowk }}$ interactions 31. In this work, we discuss results for the Brueckner-Hartree-Fock (BHF) 26] approximation of the BBG theory and for the SCGF approach.

Unfortunately, whatever realistic two-nucleon force $(2 \mathrm{NF})$ is used in a non-relativistic many-body calculation, the saturation properties of nuclear matter fail to be reproduced. Saturation densities are too large and saturation energies too attractive, with calculations falling in the so-called Coester band 32. Three-body forces (3BF) are expected to take care of this limitation. 3BFs are also required in light nuclei, whose binding energies are not correctly predicted when computed with $2 \mathrm{NF}$ only 33 . In this work, we employ the Argonne V18 (AV18) NN potential 34 in all calculations. Moreover, BHF calculations have been supplemented with the Urbana IX 3BF, reduced to a two-body density-dependent force by averaging over the third nucleon in the medium 35. The extension of the SCGF formalism to include 3BFs has been achieved only recently 36.

In the following, we report microscopic calculations of the nuclear symmetry energy and its density dependence 37. We also explore the different effect of NN correlations on SNM and PNM. We discuss how the isospin dependence of NN correlations affects the symmetry energy. To this end, we study the contribution of the different terms in the NN interaction, particularly the tensor one, to the symmetry energy 38. We describe how NN correlations produce high-momentum components and how these affect the kinetic energy and the symmetry energy 39. As mentioned above, the calculations are performed in the framework of BHF and SCGF approaches, which are well suited for this type of analysis.

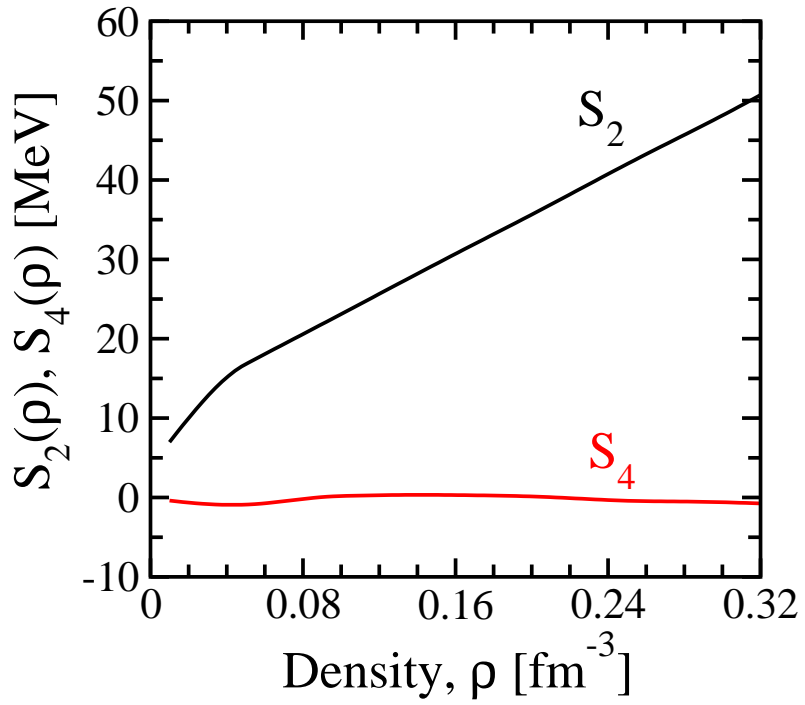

Fig. 1. (Color online) Density dependence of the symmetry energy coefficients $S_{2}$ and $S_{4}$ calculated in the BHF approximation using the AV18 interaction plus a $3 \mathrm{BF}$ of the Urbana type, as indicated in the text.

\section{Isospin asymmetric nuclear matter}

Assuming charge symmetry for nuclear forces, the energy per particle of asymmetric nuclear matter can be expanded around SNM in the isospin asymmetry parameter, $\beta=(N-Z) /(N+Z)=\left(\rho_{n}-\rho_{p}\right) / \rho$ only in terms of even powers of $\beta$ :

$$
\frac{E}{A}(\rho, \beta)=E_{S N M}(\rho)+S_{2}(\rho) \beta^{2}+S_{4}(\rho) \beta^{4}+\mathcal{O}(6) .
$$

Here, $E_{S N M}(\rho)$ is the energy per particle of SNM, $S_{2}(\rho)$ is identified (neglecting surface contributions 6, 13) with the usual symmetry energy in the semiempirical mass formula

$$
S_{2}(\rho)=\left.\frac{1}{2} \frac{\partial^{2} E / A}{\partial \beta^{2}}\right|_{\beta=0},
$$

and

$$
S_{4}(\rho)=\left.\frac{1}{24} \frac{\partial^{4} E / A}{\partial \beta^{4}}\right|_{\beta=0} .
$$

The dominant dependence of the energy per particle of asymmetric nuclear matter on $\beta$ is essentially quadratic 25, 40,41. Therefore, contributions from $S_{4}$ and other higher terms can be neglected. One can then estimate the symmetry energy by subtracting the energy per particle of PNM and that of SNM, according to

$$
S_{2}(\rho) \sim \frac{E}{A}(\rho, 1)-\frac{E}{A}(\rho, 0) .
$$

To check this approximation, we plot in Fig. 1 the density dependence of the coefficients $S_{2}$ and $S_{4}$ obtained in our BHF calculation. As expected, the coefficient $S_{4}$ is very small and $S_{2}(\rho)$ is an increasing function of $\rho$ in the density region considered $\left(0-0.3 \mathrm{fm}^{-3}\right)$. In other words, the 

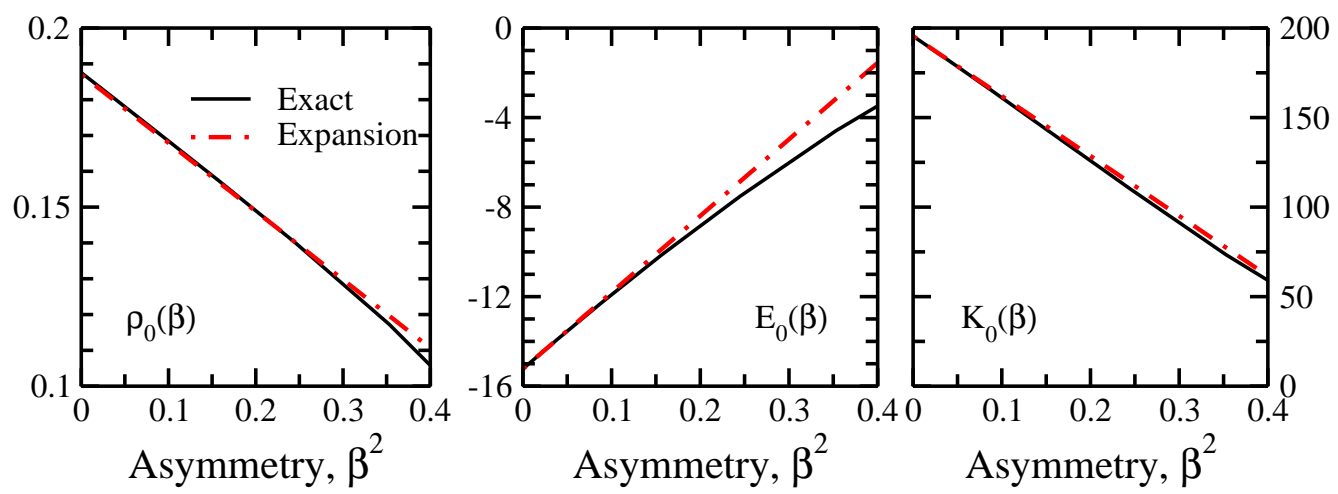

Fig. 2. (Color online) Isospin asymmetry dependence of the density (left panel), energy per particle (middle panel), and incompressibility coefficient (right panel) at the saturation point of asymmetric nuclear matter. Solid lines show the results of the BHF calculation whereas dashed lines indicate the results of the expansion of Eqs. (12), (13) and (14). Units of $E_{0}(\beta)$ and $K_{0}(\beta)$ are given in $\mathrm{MeV}$, whereas $\rho_{0}(\beta)$ is given in $\mathrm{fm}^{-3}$.

energy per particle of PNM is always larger than that of SNM and no isospin instability shows up [42.

To characterize the density dependence of the symmetry energy around saturation, it is useful to perform a series expansion in terms of the density. To this end, one considers first the density dependence of the energy per particle of SNM around the saturation density $\rho_{0}$ in terms of a few bulk parameters,

$E_{S N M}(\rho)=E_{0}+\frac{K_{0}}{2}\left(\frac{\rho-\rho_{0}}{3 \rho_{0}}\right)^{2}+\frac{Q_{0}}{6}\left(\frac{\rho-\rho_{0}}{3 \rho_{0}}\right)^{3}+\mathcal{O}(4)$.

The coefficients $E_{0}, K_{0}$ and $Q_{0}$ correspond to the energy per particle, the incompressibility coefficient, and the third derivative of the energy of SNM at saturation,

$$
E_{0}=E_{S N M}\left(\rho=\rho_{0}\right), \quad K_{0}=\left.9 \rho_{0}^{2} \frac{\partial^{2} E_{S N M}(\rho)}{\partial \rho^{2}}\right|_{\rho=\rho_{0}},
$$

and

$$
Q_{0}=\left.27 \rho_{0}^{3} \frac{\partial^{3} E_{S N M}(\rho)}{\partial \rho^{3}}\right|_{\rho=\rho_{0}} .
$$

Similarly, the symmetry energy around saturation can also be characterized in terms of a few parameters,

$$
\begin{aligned}
S_{2}(\rho) & =E_{\text {sym }}+L\left(\frac{\rho-\rho_{0}}{3 \rho_{0}}\right)+\frac{K_{\text {sym }}}{2}\left(\frac{\rho-\rho_{0}}{3 \rho_{0}}\right)^{2} \\
& +\frac{Q_{\text {sym }}}{6}\left(\frac{\rho-\rho_{0}}{3 \rho_{0}}\right)^{3}+\mathcal{O}(4)
\end{aligned}
$$

where $E_{\text {sym }}$ is the symmetry energy at saturation, and the quantities $L, K_{\text {sym }}$ and $Q_{\text {sym }}$ are related to its slope, curvature, and third derivative, at saturation density,

$$
L=\left.3 \rho_{0} \frac{\partial S_{2}(\rho)}{\partial \rho}\right|_{\rho=\rho_{0}}, \quad K_{\text {sym }}=\left.9 \rho_{0}^{2} \frac{\partial^{2} S_{2}(\rho)}{\partial \rho^{2}}\right|_{\rho=\rho_{0}},
$$

and

$$
Q_{s y m}=\left.27 \rho_{0}^{3} \frac{\partial^{3} S_{2}(\rho)}{\partial \rho^{3}}\right|_{\rho=\rho_{0}}
$$

Combining the expansions of Eqs. (11), (5) and (8), one can predict the existence of a saturation density, satisfying a zero pressure condition. For a given asymmetry, the energy per particle can be expanded around the new, asymmetry-dependent saturation density, $\rho_{0}(\beta) \sim$ $\rho_{0}\left(1-3\left(L / K_{0}\right) \beta^{2}\right)$, as

$$
\begin{aligned}
\frac{E}{A}(\rho, \beta) & =E_{0}(\beta)+\frac{K_{0}(\beta)}{2}\left(\frac{\rho-\rho_{0}(\beta)}{3 \rho_{0}(\beta)}\right)^{2} \\
& +\frac{Q_{0}(\beta)}{6}\left(\frac{\rho-\rho_{0}(\beta)}{3 \rho_{0}(\beta)}\right)^{3}+\mathcal{O}(4),
\end{aligned}
$$

where the coefficients $E_{0}(\beta), K_{0}(\beta)$, and $Q_{0}(\beta)$ define the energy per particle, the incompressibility coefficient, and the third derivative at the new saturation density, $\rho_{0}(\beta)$. These coefficients can be written in terms of the quantities defined at $\rho_{0}$, i.e., the saturation density for $\beta=0$ :

$$
\begin{gathered}
E_{0}(\beta)=E_{0}+E_{\text {sym }} \beta^{2}+\mathcal{O}(4), \\
K_{0}(\beta)=K_{0}+\left(K_{\text {sym }}-6 L-\frac{Q_{0}}{K_{0}} L\right) \beta^{2}+\mathcal{O}(4), \\
Q_{0}(\beta)=Q_{0}+\left(Q_{\text {sym }}-9 L \frac{Q_{0}}{K_{0}}\right) \beta^{2}+\mathcal{O}(4) .
\end{gathered}
$$

In Fig. 2, we explore the behavior of the saturation density $\rho_{0}(\beta)$ (left panel), energy per particle $E_{0}(\beta)$ (middle panel) and incompressibility $K_{0}(\beta)$ (right panel) as a function of $\beta^{2}$, up to an asymmetry of $\beta \sim 0.6$, for which one still finds a saturation density. The figure, shows the very good agreement between the expansion up to second order in $\beta$ (dashed lines) and the exact numerical calculations (solid lines). For $\beta=0$, one recovers the results of SNM. As $\beta$ increases, however, the saturation density, the binding energy, and the incompressibility decrease.

We finish this section by showing in Fig. 3 the density dependence of the symmetry energy $S_{2}(\rho)$ and its slope parameter, $L$, defined in Eq. (9), as obtained in our BHF calculation. 


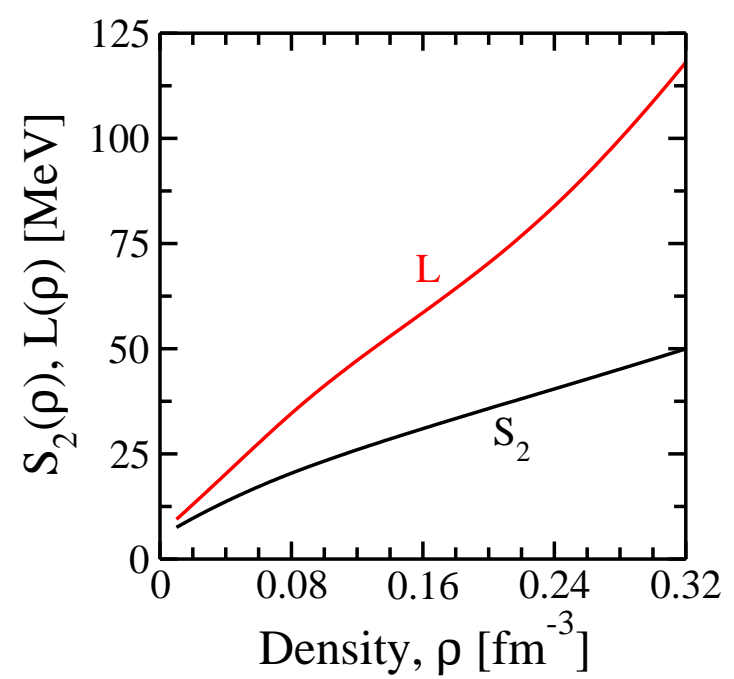

Fig. 3. (Color online) Density dependence of the symmetry energy and the slope parameter $L$ calculated in the BHF approximation using the AV18 interaction plus a 3BF of Urbana type as indicated in the text.

\section{The tensor component of the NN interaction}

Realistic NN interactions should fulfill a minimum set of requirements. In particular, realistic potentials are built to reproduce the Nijmegen database [43, which contains a full set of NN elastic scattering phase shifts up to energies of about $350 \mathrm{MeV}$, with and accuracy of $\chi^{2} / N_{\text {data }} \sim 1$. Only potentials that fulfill this condition should be used as input for the so-called ab initio many-body schemes, which aim at providing a first-principles description of the equation of state (EoS) of PNM and SNM. The Argonne V18 potential [34] is one such realistic interaction, that has been used for $a b$ initio calculations in nuclear matter and finite nuclei with a diversity of many-body approaches 44].

Applying symmetry arguments, the strong interaction part of the AV18 potential can be expressed as a sum of 18 operators,

$$
V_{i j}=\sum_{p=1,18} v_{p}\left(r_{i j}\right) O_{i j}^{p}
$$

The first 14 operators are associated to the spin, isospin, tensor, spin-orbit, and quadratic spin-orbit components of the nuclear force:

$$
\begin{aligned}
O_{i j}^{p=1, \ldots, 14}= & 1, \boldsymbol{\tau}_{i} \cdot \boldsymbol{\tau}_{j}, \boldsymbol{\sigma}_{i} \cdot \boldsymbol{\sigma}_{j},\left(\boldsymbol{\tau}_{i} \cdot \boldsymbol{\tau}_{j}\right)\left(\boldsymbol{\sigma}_{i} \cdot \boldsymbol{\sigma}_{j}\right), \\
& S_{i j}, S_{i j}\left(\boldsymbol{\tau}_{i} \cdot \boldsymbol{\tau}_{j}\right), \mathbf{L} \cdot \mathbf{S}, \mathbf{L} \cdot \mathbf{S}\left(\boldsymbol{\tau}_{i} \cdot \boldsymbol{\tau}_{j}\right), \\
& L^{2}, L^{2}\left(\boldsymbol{\tau}_{i} \cdot \boldsymbol{\tau}_{j}\right), L^{2}\left(\boldsymbol{\sigma}_{i} \cdot \boldsymbol{\sigma}_{j}\right), \\
& L^{2}\left(\boldsymbol{\tau}_{i} \cdot \boldsymbol{\tau}_{j}\right)\left(\boldsymbol{\sigma}_{i} \cdot \boldsymbol{\sigma}_{j}\right), \\
& (\mathbf{L} \cdot \mathbf{S})^{2},(\mathbf{L} \cdot \mathbf{S})^{2}\left(\boldsymbol{\tau}_{i} \cdot \boldsymbol{\tau}_{j}\right) .
\end{aligned}
$$

The four additional operators,

$$
O_{i j}^{p=15, . ., 18}=T_{i j}, T_{i j}\left(\boldsymbol{\sigma}_{i} \boldsymbol{\sigma}_{j}\right), T_{i j} S_{i j},\left(\tau_{z i}+\tau_{z j}\right),
$$

Table 1. Deuteron D-state probability $P_{D}$, quadrupole moment $Q_{d}\left(\right.$ in $\left.\mathrm{fm}^{2}\right)$, total binding energy, kinetic and potential energy, and their decomposition (in the second row) in partial waves, calculated for the AV18 NN interaction. All energies are given in $\mathrm{MeV}$.

\begin{tabular}{lllll}
\hline$P_{D}(\%)$ & $Q_{d}$ & $E$ & $K$ & $V$ \\
\hline \hline 5.78 & 0.27 & -2.24 & 19.86 & -22.10 \\
\hline & & & & \\
\hline$K_{S}$ & $K_{D}$ & $V_{S}$ & $V_{D}$ & $2 V_{S D}$ \\
\hline \hline 11.30 & 8.56 & -3.95 & 0.77 & -18.91 \\
\hline
\end{tabular}

where $T_{i j}=3 \tau_{z i} \tau_{z j}-\tau_{i} \tau_{j}$ is the isotensor operator, break charge independence. The radial functions that multiply each operator are adjusted by fitting experimental data on two-body scattering phase shifts as well as deuteron properties.

The tensor component of the NN interaction, which is crucial in the generation of NN correlations in the nuclear medium, plays a central role in the reproduction of the experimental phase shifts. Moreover, the tensor is also largely responsible for the structure and binding energy of the deuteron, the simplest bound nucleonic system. Even though the deuteron only probes the NN potential in the ${ }^{3} S_{1}-{ }^{3} D_{1}$ partial waves, the analysis of the independent contributions of the different waves $(S-, D-$ and mixed channel) highlights the importance of the different components of the NN interaction [44,45]. The first observation is that for a realistic potential, as AV18, the binding energy of the deuteron results from a strong cancellation between the kinetic and the interaction energies (see first row of Table 1). For the AV18, the binding energy, $E=-2.24$ $\mathrm{MeV}$, comes from a large kinetic, $K=19.86 \mathrm{MeV}$, and interaction, $V=-22.10 \mathrm{MeV}$, energies. Note that this is the binding energy obtained with only the strong interaction part of AV18, i.e., when all small electromagnetic terms are omitted. These repulsive electromagnetic terms shift the binding energy to the true experimental value $E=-2.22 \mathrm{MeV}$. It is also worth stressing that the charge-dependent terms of $V_{p}(p=15, \ldots, 18)$, described in terms of an isotensor operator, have no contribution in the isosinglet deuteron state.

The $D$-state probability as well as the quadrupole moment are also a direct consequence of the tensor component of the nuclear force that allows for the coupling between the $S$ and the $D$ wave. It is instructive to separate the contributions of the ${ }^{3} S_{1}$ and ${ }^{3} D_{1}$ states to the total kinetic and potential energies (see second row of Table 1). Assuming that the deuteron is a properly normalized combination of the ${ }^{3} S_{1}$ and ${ }^{3} D_{1}$ partial waves, we define the contributions of the $S$ and $D$ states to the kinetic energy, $K_{S}=\left\langle{ }^{3} S_{1}|K|^{3} S_{1}\right\rangle$ and $K_{D}=\left\langle{ }^{3} D_{1}|K|^{3} D_{1}\right\rangle$, and to the potential energy, $V_{S}=\left\langle{ }^{3} S_{1}|V|^{3} S_{1}\right\rangle$ and $V_{D}=$ $\left\langle{ }^{3} D_{1}|V|^{3} D_{1}\right\rangle$. The latter also receives a contribution from the ${ }^{3} S_{1}-{ }^{3} D_{1}$ mixing, $V_{S D}=\left\langle{ }^{3} S_{1}|V|^{3} D_{1}\right\rangle$. Actually the largest contribution comes from the mixing term, $V_{S D}$, 
Table 2. Contribution of the different components of the NN AV18 interaction to the binding energy of the deuteron. All energies are given in $\mathrm{MeV}$.

\begin{tabular}{llll}
\hline Central & Tensor & Spin-orbit & $L^{2}$ \\
\hline \hline-4.45 & -16.62 & -3.75 & 2.72 \\
\hline
\end{tabular}

which accounts for more than $85 \%$ of the final value of the potential energy.

Additional information can be obtained by looking at the expectation values of the different components of the potential on the ground-state wave function of the deuteron. We have grouped the 18 components into four sets: the first four operators $(p=1, \ldots, 4)$, the tensor components, $S_{i j}(p=5,6)$, the spin-orbit components $(p=7,8$ and $p=13,14)$, and the quadratic orbital angular momentum components $L^{2}(p=9, \ldots, 12)$. The group of chargedependent terms, $p=15, \ldots, 18$, does not contribute to the deuteron. The results of this decomposition are presented in Table 2, As expected, the largest contribution corresponds to the tensor component. All contributions are attractive, except the one proportional to $L^{2}$, which is slightly repulsive. Notice also that the spin-orbit contribution is non-negligible and amounts to $17 \%$ of the total potential energy.

\section{Brueckner-Hartree-Fock results}

The energy per particle of asymmetric nuclear matter in the BHF approach is calculated as

$$
\frac{E}{A}(\rho, \beta)=\frac{1}{A} \sum_{\tau} \sum_{|k|<k_{F_{\tau}}}\left(\frac{\hbar^{2} k^{2}}{2 m}+\frac{1}{2} R e\left[U_{\tau(k)}\right]\right),
$$

where $U_{\tau}(k)$ represents the mean field "felt" by a nucleon $(\tau=n, p)$ due to its interaction with the other nucleons of the medium. $U_{\tau}(k)$ is calculated through the "on-shell energy" $G$ matrix,

$U_{\tau}=\sum_{\tau^{\prime}} \sum_{|k|<k_{F_{\tau^{\prime}}}}\left\langle\boldsymbol{k} \boldsymbol{k}^{\prime}\left|G_{\tau \tau^{\prime} ; \tau \tau^{\prime}}\left(\omega=\epsilon_{\tau}(k)+\epsilon_{\tau^{\prime}}\left(k^{\prime}\right)\right)\right| \boldsymbol{k} \boldsymbol{k}^{\prime}\right\rangle_{A}$

where the sum runs over all neutron and proton occupied states, and the matrix elements are properly antisymmetrized. The single particle energy, $\epsilon_{\tau}(k)$, is defined in terms of the single-particle kinetic energy and the singleparticle potential,

$$
\epsilon_{\tau}(k)=\frac{\hbar^{2} k^{2}}{2 m_{\tau}}+\operatorname{Re}\left[U_{\tau}(k)\right] .
$$

Finally, the $G$-matrices describing the effective interaction between two nucleons in the medium are constructed by
Table 3. Bulk parameters characterizing the density dependence of the energy of SNM and the symmetry energy around the saturation density for our BHF calculation with and without $3 \mathrm{BF}$. All quantities are in $\mathrm{MeV}$, except $\rho_{0}$, given in $\mathrm{fm}^{-3}$.

\begin{tabular}{lllll}
\hline & $\rho_{0}$ & $E_{0}$ & $K_{0}$ & $Q_{0}$ \\
\hline \hline BHF (no 3BF) & 0.240 & -17.30 & 213.6 & -225.1 \\
BHF (3BF) & 0.187 & -15.23 & 195.5 & -280.9 \\
\hline & $E_{\text {sym }}$ & $L$ & $K_{\text {sym }}$ & $Q_{\text {sym }}$ \\
\hline \hline BHF (no 3BF) & 35.8 & 63.1 & -27.8 & -159.8 \\
BHF (3BF) & 34.3 & 66.5 & -31.3 & -112.8 \\
\hline
\end{tabular}

solving the Bethe-Goldstone equation

$$
\begin{aligned}
& G_{\tau_{1} \tau_{2} ; \tau_{3} \tau_{4}}(\omega)=V_{\tau_{1} \tau_{2} ; \tau_{3} \tau_{4}} \\
&+\sum_{j k} V_{\tau_{1} \tau_{2} ; \tau_{j} \tau_{k}} \frac{Q_{\tau_{j} \tau_{k}}}{\omega-\epsilon_{j}-\epsilon_{k}+i \eta} G_{\tau_{j} \tau_{k} ; \tau_{3} \tau_{4}}(\omega)
\end{aligned}
$$

where $V$ denotes the free-space NN interaction, $Q_{\tau_{j} \tau_{k}}$ is the Pauli operator which allows only for intermediate states compatible with the Pauli principle, and $\omega$ is the so-called starting energy, which corresponds to the sum of nonrelativistic energies of the interacting nucleons. Note that the whole procedure requires a self-consistent process. It has been shown by Song et al., 46 that the contribution to the energy from three-hole-line diagrams (that account for the effect of three-body correlations) is minimized when the so-called continuous prescription [47] is adopted for the in-medium potential, which is a strong indication of the convergence of the BBG expansion. We adopt this prescription in our calculation.

The BHF calculations discussed in this work have been performed with the realistic AV18 NN interaction supplemented with a $3 \mathrm{BF}$ of Urbana type. This $3 \mathrm{BF}$ contains two parameters that are fixed by requiring that the BHF calculation reproduces the energy and saturation density of SNM. The results reported here correspond to the original set of parameters of Baldo and Ferreira in Ref. 35. Moreover, the $3 \mathrm{BF}$ has been reduced to a two-body densitydependent force by averaging over the third nucleon in the medium 35. See also Refs. 48, 49,50 for an extensive analysis of the use of 3BFs in nuclear and neutron matter.

We start the discussion of the BHF results by showing in Table 3 the bulk parameters characterizing the density dependence of the energy of SNM and the symmetry energy around saturation density. We report the BHF results obtained with and without three-body forces. The comparison of the different quantities is strongly influenced by the fact that they are calculated at a value of the saturation density which is different with and without $3 \mathrm{BF}$. Note that, in general, the effects of the $3 \mathrm{BF}$ are more important on the isoscalar properties, like $K_{0}$. Our BHF calculation gives a value of $L=66.5 \mathrm{MeV}$, compatible with recent experimental constraints (see e.g., Fig. 1 of Ref. [51]). 
Table 4. Free-Fermi gas contribution, $\Delta E_{B H F}$ and total energy per particle of PNM and SNM. The respective contributions to $E_{\text {sym }}$ and $L$ are also reported. The results correspond to the saturation density of the BHF approach, $\rho_{0}=0.187$ $\mathrm{fm}^{-3}$ for the AV18+3BF. All results are given in $\mathrm{MeV}$

\begin{tabular}{lllll}
\hline & $E_{P N M}$ & $E_{S N M}$ & $E_{\text {sym }}$ & $L$ \\
\hline \hline FFG & 38.911 & 24.529 & 14.382 & 28.779 \\
$\Delta E_{B H F}$ & -19.682 & -39.600 & 19.918 & 37.721 \\
Total & 19.229 & -15.071 & 34.300 & 66.500 \\
\hline
\end{tabular}

The properties associated with the density dependence of the symmetry energy are little affected by the $3 \mathrm{BF}$. This is due to both a shift in saturation density and to a similarly repulsive effect on the energy of both SNM and PNM once the density shift is taken into account. Overall, there is a small dependence of the isovector properties on the $3 \mathrm{BF}$, even though the independent contributions on SNM and PNM are not small. Recently, the importance of $3 \mathrm{BF}$ s has been revisited in the context of chiral perturbation theory 52. New fitting protocols of two-nucleon forces seem to indicate that the effect of $3 \mathrm{BF}$ could actually be rather small in PNM. Nevertheless, the relative importance of the two- and three-body contributions change with the resolution scale. It is not clear that such observations, valid for the somewhat soft chiral interactions, apply when considering a hard interaction like AV18. In any case, one does not expect isovector properties to depend much on the presence or absence of 3BFs.

In the BHF approach, one calculates the correction, $\Delta E_{B H F}$, to the energy of the free Fermi gas (FFG), i.e., the underlying non-interacting system, and expresses the total energy as

$$
E_{B H F}=E_{F F G}+\Delta E_{B H F} .
$$

In Table 4, we report this decomposition for the energy per particle of SNM and PNM, at the saturation density provided by the AV18+3BF calculation. The symmetry energy is calculated as the difference of the total energy per particle of PNM and SNM. The FFG energy is larger for neutron matter than for symmetric matter and therefore its contribution to the symmetry energy is positive and amounts to $\sim 14.38 \mathrm{MeV} . \Delta E_{B H F}$ is less attractive for neutron matter than for nuclear matter and also gives a positive contribution $(\sim 19.92 \mathrm{MeV})$ to the symmetry energy. The addition of these two quantities, which are of the same order, provides a symmetry energy of $\sim 34.3$ $\mathrm{MeV}$. The contributions to $L$ can be decomposed similarly, but in this case the contribution of $\Delta E_{B H F}(37.22 \mathrm{MeV})$ is slightly larger than that of the FFG, which amounts to $28.78 \mathrm{MeV}$.

To get a further physical insight into $\triangle E_{B H F}$, it is useful to look at its spin-isospin $(S, T)$ decomposition, reported in Table 5. As expected, the main contribution is from the $(1,0)$ channel which is acting only in SNM and has a large attractive contribution. It is precisely in this
Table 5. Spin-isospin (S,T) channel decomposition of $\Delta E_{B H F}$ for PNM and SNM. The respective contributions to $E_{\text {sym }}$ and $L$ are also reported. The results correspond to the saturation density of the BHF approach, $\rho_{0}=0.187 \mathrm{fm}^{-3}$ for the AV18+3BF. All results are given in $\mathrm{MeV}$

\begin{tabular}{lllll}
\hline$(\mathrm{S}, \mathrm{T})$ & $E_{P N M}$ & $E_{S N M}$ & $E_{\text {sym }}$ & $L$ \\
\hline \hline$(0,0)$ & 0 & 5.894 & -5.894 & -23.085 \\
$(0,1)$ & -21.041 & -17.764 & -3.277 & -3.142 \\
$(1,0)$ & 0 & -28.363 & 28.363 & 51.696 \\
$(1,1)$ & 1.359 & 0.633 & 0.726 & 12.252 \\
\hline
\end{tabular}

channel where the tensor component of the $\mathrm{NN}$ force is active. Note that the $T=1$ channels give similar contributions in nuclear and neutron matter and therefore its contribution to the symmetry energy is small. The channel $(0,0)$ gives a repulsive contribution to the total energy in SNM and since it does not play any role in neutron matter, its contribution to the total symmetry energy is negative. Notice again that the tensor force is not acting in this channel.

Let us further this analysis by looking at the contributions of the different partial waves to $\triangle E_{B H F}$, as shown in Table 6. Notice that the ${ }^{1} S_{0}$ contribution, which is dominated by the central component of the NN potential, has a similarly large contribution to both PNM and SNM and therefore its effect on the symmetry energy is almost negligible. The largest contribution is provided by the ${ }^{3} S_{1}-{ }^{3} D_{1}$ partial wave, which corresponds to $T=0$, active only in nuclear matter. For larger values of $J$, the contributions become smaller and many cancellations take place. In general, one observes that the final energy is the result of a large cancellation between $E_{F F G}$ and $\Delta E_{B H F}$ and that the absolute value of the correction $\triangle E_{B H F}$ for neutron matter is significantly smaller than for nuclear matter. This observation points to the well accepted fact that neutron matter is less correlated than nuclear matter.

In the case of the deuteron, the total binding energy is the result of a strong cancellation between the kinetic energy and the potential energy. The large kinetic energy is a consequence of the $\mathrm{NN}$ correlations existing in the deuteron, i.e., in the ${ }^{3} S_{1}-{ }^{3} D_{1}$ channel. In that sense, we would like to study the decomposition of the total energy of the infinite system in the kinetic and potential energy. This kinetic energy will contain the effects of correlations and will be larger than the energy of the FFG. Therefore the difference between the correlated kinetic energy and $E_{F F G}$ can quantify NN correlations.

Unfortunately, the BHF approach does not give direct access to the separate contribution of the kinetic and potential energies because it does not provide the correlated many-body wave function, $|\Psi\rangle$. However, it has been shown 53] that the Hellmann-Feynman theorem [54] can be used to estimate the ground state expectation value of the interaction energy. The kinetic energy can then be calculated simply by subtracting the expectation value of the 
Table 6. Partial wave decomposition of $\Delta E_{B H F}$ for PNM and SNM. The contributions of each partial wave to $E_{\text {sym }}$ and $L$ are also reported. The results correspond to the saturation density of the BHF approach, $\rho_{0}=0.187 \mathrm{fm}^{-3}$ for the AV18+3BF. All results are given in $\mathrm{MeV}$.

\begin{tabular}{lllll}
\hline Partial wave & $E_{P N M}$ & $E_{S N M}$ & $E_{\text {sym }}$ & $L$ \\
\hline \hline${ }^{1} S_{0}$ & -14.330 & -14.407 & 0.077 & 11.229 \\
${ }^{3} S_{1}$ & 0 & -24.865 & 24.865 & 35.521 \\
${ }^{1} P_{1}$ & 0 & 5.193 & -5.193 & -20.201 \\
${ }^{3} P_{0}$ & -4.522 & -3.713 & -0.809 & 0.224 \\
${ }^{3} P_{1}$ & 18.459 & 12.002 & 6.457 & 27.702 \\
${ }^{3} P_{2}$ & -13.550 & -8.102 & -5.448 & -17.784 \\
${ }^{1} D_{2}$ & -5.850 & -3.154 & -2.696 & -10.888 \\
${ }^{3} D_{1}$ & 0 & 1.036 & -1.036 & -3.894 \\
${ }^{3} D_{2}$ & 0 & -3.795 & 3.795 & 15.844 \\
${ }^{3} D_{3}$ & 0 & -0.522 & 0.522 & 3.305 \\
${ }^{1} F_{3}$ & 0 & 0.699 & -0.699 & -3.394 \\
${ }^{3} F_{2}$ & -0.651 & -0.221 & -0.430 & -1.515 \\
${ }^{3} F_{3}$ & 2.022 & 0.826 & 1.196 & 5.026 \\
${ }^{3} F_{4}$ & -0.743 & -0.183 & -0.560 & -3.006 \\
${ }^{1} G_{4}$ & -0.810 & -0.247 & -0.563 & -3.029 \\
${ }^{3} G_{3}$ & 0 & 0.002 & -0.002 & 0.425 \\
${ }^{3} G_{4}$ & 0 & -0.213 & 0.213 & 0.449 \\
${ }^{3} G_{5}$ & 0 & -0.053 & 0.053 & 0.617 \\
${ }^{1} H_{5}$ & 0 & 0.029 & -0.029 & 0.122 \\
${ }^{3} H_{4}$ & 0.034 & 0.040 & -0.007 & 0.224 \\
${ }^{3} H_{5}$ & 0.226 & -0.033 & 0.258 & 0.949 \\
${ }^{3} H_{6}$ & 0.044 & 0.035 & 0.010 & 0.136 \\
\hline
\end{tabular}

Table 7. Kinetic, $\langle K\rangle$, and potential, $\langle V\rangle$, contributions to $E_{P N M}, E_{S N M}, E_{\text {sym }}$ and $L$. Units are given in $\mathrm{MeV}$.

\begin{tabular}{lllcl}
\hline & $E_{P N M}$ & $E_{S N M}$ & $E_{\text {sym }}$ & $L$ \\
\hline \hline$\langle K\rangle$ & 53.321 & 54.294 & -0.973 & 14.896 \\
$\langle V\rangle$ & -34.251 & -69.524 & 35.273 & 51.604 \\
Total & 19.070 & -15.230 & 34.300 & 66.500 \\
\hline
\end{tabular}

potential energy from $E_{B H F}$. Writing the nuclear hamiltonian as $H=T+V$, and defining a $\lambda$ dependent potential, $H(\lambda)=T+\lambda V$, the expectation value of the potential energy is given by:

$$
\langle V\rangle \equiv \frac{\langle\Psi|V| \Psi\rangle}{\langle\Psi \mid \Psi\rangle}=\left(\frac{d E}{d \lambda}\right)_{\lambda=1} .
$$

In Table 7 we show the kinetic and potential energy contributions to the total energy of PNM, SNM, $E_{\text {sym }}$ and $L$ at the saturation density, $\rho_{0}=0.187 \mathrm{fm}^{-3}$, provided by the AV18+3BF within the BHF approach.

As in the case of the deuteron, the total energy of both PNM and SNM are the result of a strong cancellation between the kinetic and potential energies. It is worth noticing that the kinetic energy contribution to $E_{\text {sym }}$ is very small and slightly negative. This is in contrast to the results for the FFG (see Table 4). The increase of the
Table 8. Spin-isospin (S,T) channel decomposition of the potential contribution to $E_{P N M}, E_{S N M}$ and $E_{\text {sym }}$ and $L$. The results correspond to the saturation density of the BHF approach, $\rho_{0}=0.187 \mathrm{fm}^{-3}$ for the AV18+3BF. All results are given in $\mathrm{MeV}$.

\begin{tabular}{lllll}
\hline$(\mathrm{S}, \mathrm{T})$ & $E_{P N M}$ & $E_{S N M}$ & $E_{\text {sym }}$ & $L$ \\
\hline \hline$(0,0)$ & 0 & 5.6 & -5.6 & -21.457 \\
$(0,1)$ & -29.889 & -23.064 & -6.825 & -17.950 \\
$(1,0)$ & 0 & -49.836 & 49.836 & 90.561 \\
$(1,1)$ & -4.362 & -2.224 & -2.138 & 0.450 \\
\hline
\end{tabular}

kinetic energy with respect to the FFG energy, which is due mainly to short range and tensor correlations, is much larger for SNM than for PNM. Again this is an indication that, at the same density, SNM is more correlated than PNM.

It is also worth mentioning that the kinetic contribution to $L$ is smaller than the corresponding one of the FFG $\left(L_{F F G} \sim 29.2 \mathrm{MeV}\right)$ reported in Table 4. Clearly the major contribution to both $E_{\text {sym }}$ and $L$ is due to the potential energy part. Note that this contribution is practically equal to the total value of $E_{\text {sym }}$ and it represents $\sim 78 \%$ of $L$. Results along these lines have been recently reported by $\mathrm{Xu}$ and $\mathrm{Li}$ [55] using a phenomenological model for $n(k)$.

The spin-isospin $(S, T)$ channel decomposition of the potential part of $E_{P N M}, E_{S N M}, E_{\text {sym }}$ and $L$ is also illustrative. This is reported in Table 8 at $\rho_{0}$. As in the case of $\Delta E_{B H F}$, the largest contribution to both $E_{\text {sym }}$ and $L$ is provided by the $(S=1, T=0)$ channel, which is where the tensor is active. Interestingly, the $S=0$ channels have a small and similar negative contribution to $E_{\text {sym }}$ and also a moderate negative contribution to $L$ showing in total a strong cancellation with the contribution of the channel $(S=1, T=0)$. However, the origin of these contributions is qualitatively different. While the channel $(S=0$, $T=0$ ) does not contribute to neutron matter and has a small repulsive contribution to nuclear matter, the contribution of the channel $(S=0, T=1)$ is the result of a strong cancellation of large attractive contributions of this channel in both PNM and SNM. Analogous conclusions can be obtained from Table 9, where the partial wave decomposition of the potential energy is reported. Note that similar arguments have been already pointed out by other authors $25,42,55,56,57,58,59,60,61,62,63,64,65]$.

Next, we analyze the contribution to the potential energy of the different components of the nuclear force. To such end, we apply the Hellmann-Feynman theorem to the separate components of the AV18 potential and the Urbana IX three-body force. The results are reported in Table 10, The central contribution $\left\langle V_{1}\right\rangle$ is large, attractive and similar for neutron and nuclear matter and therefore gives a small contribution to $E_{\text {sym }}$. The largest contribution to isovector properties is from the tensor $\left\langle V_{S_{i j}\left(\boldsymbol{\tau}_{i} \boldsymbol{\tau}_{j}\right)}\right\rangle$, which acts very efficiently to supply attraction in SNM. 
Table 9. Partial wave decomposition of the potential part of $E_{P N M}, E_{S N M}, E_{s y m}$ and $L$. The results correspond to the saturation density of the BHF approach, $\rho_{0}=0.187 \mathrm{fm}^{-3}$ for the AV18+3BF. Units are given in $\mathrm{MeV}$.

\begin{tabular}{lllll}
\hline Partial wave & $E_{P N M}$ & $E_{S N M}$ & $E_{\text {sym }}$ & $L$ \\
\hline \hline${ }^{1} S_{0}$ & -23.070 & -19.660 & -3.410 & -3.459 \\
${ }^{3} S_{1}$ & 0 & -45.810 & 45.810 & 71.855 \\
${ }^{1} P_{1}$ & 0 & 4.904 & -4.904 & -18.601 \\
${ }^{3} P_{0}$ & -5.312 & -4.029 & -1.292 & -1.898 \\
${ }^{3} P_{1}$ & 16.110 & 10.720 & 5.390 & 21.9149 \\
${ }^{3} P_{2}$ & -16.000 & -9.334 & -6.666 & -21.168 \\
${ }^{1} D_{2}$ & -5.956 & -3.201 & -2.755 & -11.033 \\
${ }^{3} D_{1}$ & 0 & 0.981 & -0.981 & -3.739 \\
${ }^{3} D_{2}$ & 0 & -3.982 & 3.982 & 16.601 \\
${ }^{3} D_{3}$ & 0 & -0.798 & 0.798 & 4.895 \\
${ }^{1} F_{3}$ & 0 & 0.694 & -0.694 & -3.348 \\
${ }^{3} F_{2}$ & -0.695 & -0.229 & -0.466 & -1.799 \\
${ }^{3} F_{3}$ & 2.000 & 0.821 & 1.179 & 4.883 \\
${ }^{3} F_{4}$ & -0.796 & -0.194 & -0.602 & -3.239 \\
${ }^{1} G_{4}$ & -0.812 & -0.247 & -0.565 & -3.036 \\
${ }^{3} G_{3}$ & 0 & -0.001 & 0.001 & 0.441 \\
${ }^{3} G_{4}$ & 0 & -0.213 & 0.213 & 0.449 \\
${ }^{3} G_{5}$ & 0 & -0.057 & 0.057 & 0.650 \\
${ }^{1} H_{5}$ & 0 & 0.029 & -0.029 & 0.107 \\
${ }^{3} H_{4}$ & 0.033 & 0.040 & -0.007 & 0.232 \\
${ }^{3} H_{5}$ & 0.225 & -0.033 & 0.258 & 0.968 \\
${ }^{3} H_{6}$ & 0.043 & 0.034 & 0.009 & 0.144 \\
\hline
\end{tabular}

As mentioned above, the Urbana IX 3BF is reduced to an effective density-dependent two-body force when used in the BHF approach. This reduced, effective two-body force contains three components of the type $u_{p}\left(r_{i j}, \rho\right) O_{i j}^{p}$ where $O_{i j}^{p=1,3}=1,\left(\boldsymbol{\sigma}_{i} \cdot \boldsymbol{\sigma}_{j}\right)\left(\boldsymbol{\tau}_{i} \cdot \boldsymbol{\tau}_{j}\right), S_{i j}\left(\boldsymbol{\tau}_{i} \cdot \boldsymbol{\tau}_{j}\right)$. This introduces additional central, $\sigma \tau$ and tensor terms, which are reported on the last three rows of Table 10 (see e.g., Ref. 35 for details). The contribution of the two-body density dependent effective force to $E_{\text {sym }}$ can be considered small, with the tensor component being the most important. These results clearly confirm that the tensor force gives the largest contribution to both $E_{\text {sym }}$ and $L$. The contributions from the other components are either negligible, or almost cancel out.

\section{High-momentum components in the symmetry energy}

In the previous section, we have used the kinetic energy difference between the correlated system and the underlying FFG as a measure of NN correlations. The kinetic energy is the result of integrating the momentum distribution weighted with the kinetic energy associated to each momentum. The presence of correlations modifies the step function associated to the FFG, $\Theta\left(k_{F}-k\right)$. There is a promotion of particles to higher momentum states and, as a consequence, there is also a depletion below $k_{F}$. Thus, the
Table 10. Contributions to $E_{P N M}, E_{S N M}, E_{\text {sym }}$ and $L$ from the different components of the AV18 potential (indicated as $\left.\left\langle V_{i}\right\rangle\right)$ and the reduced Urbana three-body force (indicated as $\left.\left\langle U_{i}\right\rangle\right)$. The results correspond to the saturation density of the BHF approach, $\rho_{0}=0.187 \mathrm{fm}^{-3}$ for the AV18+3BF. Units are given in $\mathrm{MeV}$.

\begin{tabular}{|c|c|c|c|c|}
\hline$\langle V\rangle$ & $E_{P N M}$ & $E_{S N M}$ & $E_{\text {sym }}$ & $L$ \\
\hline$\left\langle V_{1}\right\rangle$ & -31.212 & -32.710 & 1.498 & -5.580 \\
\hline$\left\langle V_{\boldsymbol{\tau}_{i}} \boldsymbol{\tau}_{j}\right\rangle$ & -4.957 & 3.997 & -8.954 & -20.383 \\
\hline$\left\langle V_{\boldsymbol{\sigma}_{i} \boldsymbol{\sigma}_{j}}\right\rangle$ & -0.319 & -0.382 & 0.063 & 2.392 \\
\hline$\left\langle V_{\left(\boldsymbol{\tau}_{i} \boldsymbol{\tau}_{j}\right)\left(\boldsymbol{\sigma}_{i} \boldsymbol{\sigma}_{i}\right)}\right\rangle$ & -5.724 & -11.388 & 5.664 & 2.521 \\
\hline$\left\langle V_{S_{i j}}\right\rangle$ & -0.792 & 1.912 & -2.704 & -4.998 \\
\hline$\left\langle V_{S_{i j}\left(\boldsymbol{\tau}_{j} \boldsymbol{\tau}_{j}\right)}\right\rangle$ & -4.989 & -37.592 & 32.603 & 47.095 \\
\hline$\left\langle V_{\boldsymbol{L} \boldsymbol{S}}\right\rangle$ & -7.538 & -1.754 & -5.784 & -12.251 \\
\hline$\left\langle V_{\boldsymbol{L}} \boldsymbol{S}\left(\boldsymbol{\tau}_{i} \boldsymbol{\tau}_{j}\right)\right\rangle$ & -2.671 & -6.539 & 3.868 & 3.969 \\
\hline$\left\langle V_{L^{2}}\right\rangle$ & 11.850 & 13.610 & -1.760 & 1.521 \\
\hline$\left\langle V_{L^{2}\left(\boldsymbol{\tau}_{i} \boldsymbol{\tau}_{j}\right)}\right\rangle$ & -2.788 & 0.270 & -3.058 & -14.262 \\
\hline$\left\langle V_{L^{2}\left(\boldsymbol{\sigma}_{i} \boldsymbol{\sigma}_{i}\right)}\right\rangle$ & 1.265 & 1.383 & -0.118 & 1.405 \\
\hline$\left\langle V_{L^{2}\left(\boldsymbol{\sigma}_{i} \boldsymbol{\sigma}_{i}\right)\left(\boldsymbol{\tau}_{i} \boldsymbol{\tau}_{j}\right)}\right\rangle$ & 0.051 & 0.008 & 0.043 & -0.341 \\
\hline$\left\langle V_{(\boldsymbol{L} \boldsymbol{S})^{2}}\right\rangle$ & 4.194 & 5.682 & -1.488 & -0.327 \\
\hline$\left\langle V_{(\boldsymbol{L} \boldsymbol{S})^{2}\left(\boldsymbol{\tau}_{i} \boldsymbol{\tau}_{j}\right)}\right\rangle$ & 5.169 & -6.190 & 11.359 & 31.368 \\
\hline$\left\langle V_{T_{i j}}\right\rangle$ & 0.003 & 0.039 & -0.036 & -0.022 \\
\hline$\left\langle V_{T_{i j}}\left(\boldsymbol{\sigma}_{i} \boldsymbol{\sigma}_{j}\right)\right\rangle$ & -0.017 & -0.106 & 0.089 & 0.042 \\
\hline$\left\langle V_{T_{i j} S_{i j}}\right\rangle$ & 0.004 & 0.079 & -0.075 & -0.124 \\
\hline$\left\langle V_{\left(\tau_{z_{i}}+\tau_{z_{j}}\right)}\right\rangle$ & -0.084 & -0.001 & -0.083 & -0.331 \\
\hline$\left\langle U_{1}\right\rangle$ & 2.985 & 3.251 & -0.266 & -0.630 \\
\hline$\left\langle U_{\left(\boldsymbol{\tau}_{j} \boldsymbol{\tau}_{j}\right)\left(\boldsymbol{\sigma}_{i} \boldsymbol{\sigma}_{j}\right)}\right\rangle$ & 2.252 & 3.999 & -1.745 & -7.228 \\
\hline$\left\langle U_{S_{i j}\left(\boldsymbol{\tau}_{i} \boldsymbol{\tau}_{j}\right)}\right\rangle$ & -0.935 & -7.092 & 6.157 & 27.768 \\
\hline
\end{tabular}

single-particle momentum distributions can be taken also as a probe of the correlations embedded in the nuclear wave function.

In this section, we would like to discuss the effects of correlations directly on the momentum distributions. To this end, we rely on another microscopic many-body approach, the Self-Consistent Green's Function method. This approach provides access to the single particle properties in a natural way. In particular, $n(k)$ can be obtained and, as a consequence, the kinetic energy can be studied. We will analyze how NN correlations affect differently the momentum distribution in symmetric and neutron matter and, therefore, which is the effect of the high momentum components on the symmetry energy [39].

In the SCGF method, a diagrammatic expansion is employed to solve for the in-medium one-body propagator, rather than for the energy of the system. For infinite matter, the method is conventionally applied at the ladder approximation level. SCGF calculations give direct access to microscopic properties related to the single-particle propagator. These include self-energies, spectral functions and momentum distributions, from which one can derive microscopic and bulk properties. The ladder approximation provides a microscopic description of short-range and tensor effects via a fully dressed propagation of nucleons in nuclear matter. This is achieved by: a) computing the 
scattering of particles via a $T$-matrix (or effective interaction) in the medium, b) extracting a self-energy out of the effective interaction and, c) using the Dyson equation to build two-body propagators which are subsequently inserted in the scattering equation. To solve this closed set of equations, a self-consistency procedure is required. The formalism is well established for two-body potentials and its extension to include three-body forces has been only recently considered 66, 36. Here, all the SCGF results have been obtained with the AV18 NN interaction. Three-body forces are not included in this part of the work.

The bulk properties of nuclear matter and neutron matter are obtained within the SCGF approach through the Galitskii-Migdal-Koltun sum-rule:

$$
\frac{E}{A}=\frac{\nu}{\rho} \int \frac{d^{3} k}{(2 \pi)^{3}} \int \frac{d \omega}{2 \pi} \frac{1}{2}\left(\frac{k^{2}}{2 m}+\omega\right) \mathcal{A}(k, \omega) f(\omega),
$$

where $\nu=4(2)$ is the spin-isospin degeneracy of nuclear (neutron) matter, $\rho$ is the total density and $f(\omega)=[1+$ $\left.e^{(\omega-\mu) / T}\right]^{-1}$ is a Fermi-Dirac distribution. $\mathcal{A}(k, \omega)$ is the one-body spectral function which, loosely speaking, represents the probability of knocking out or adding a particle with a given single-particle momentum, $k$, and energy, $\omega$. The single-particle spectral function provides the full knowledge of the one-body propagator and gives access to the calculation of all the one-body properties of the system. For instance, the momentum distribution, $n(k)$, is obtained by convoluting the spectral function with a Fermi-Dirac factor:

$$
n(k)=\int \frac{d \omega}{2 \pi} \mathcal{A}(k, \omega) f(\omega) .
$$

The total density sum-rule,

$$
\rho=\nu \int \frac{d^{3} k}{(2 \pi)^{3}} n(k),
$$

is used to extract the chemical potential $\mu$. This can also be calculated using its thermodynamical definition, i.e., by a density derivative of the free-energy density. The agreement between these two determinations of the chemical potential is taken as a test of the thermodynamical consistency of the approach. Notice that, to avoid pairing instabilities, the SCGF calculations have been performed at finite temperature $(\mathrm{T}=5 \mathrm{MeV}) 67$.

As mentioned earlier, correlations beyond the meanfield approximation have a particularly clear manifestation in the momentum distribution 68. A sizable depletion appears below the Fermi sea, while high-momentum components are populated. To illustrate this point, we show in Fig. 4 the momentum distribution of SNM (left panel) and PNM (right panel), at $\rho=0.16 \mathrm{fm}^{-3}$ and $T=$ $5 \mathrm{MeV}$. The results obtained within the SCGF method for AV18 (solid lines) are compared to the momentum distributions of the FFG in the same conditions (dashed lines). The FFG is used here as a reference for the thermal effects, as deviations from the step function give a measure of the importance of finite temperature. A common characteristic of the SCGF and the FFG $n(k)$ is the softening
Table 11. Contributions of different momentum regions to the integrated strength with $m=2$ (columns 2 and 5), kinetic (3 and 6 , in $\mathrm{MeV}$ ) and total energies (4 and 7, in MeV). SNM (PNM) results are presented in columns 2, 3 and 4 (5, 6 and 7). Rows 3 to 6 show SCGF results with the AV18 NN interaction, whereas rows 7 to 10 correspond to the FFG results. All results are computed at $\rho=0.16 \mathrm{fm}^{-3}$ and $T=5 \mathrm{MeV}$.

\begin{tabular}{l|lll|lll|} 
& \multicolumn{3}{|c|}{ SNM } & \multicolumn{3}{r|}{ PNM } \\
\hline$\left(k_{i}, k_{f}\right)$ & $\phi_{2}$ & $\mathrm{~K} / \mathrm{A}$ & $\mathrm{E} / \mathrm{A}$ & $\phi_{2}$ & $\mathrm{~K} / \mathrm{A}$ & $\mathrm{E} / \mathrm{A}$ \\
\hline \hline$\left(0, k_{F}\right)$ & 0.755 & 15.6 & -7.65 & 0.863 & 28.7 & 11.6 \\
$\left(k_{F}, 2 k_{F}\right)$ & 0.194 & 11.4 & -1.00 & 0.119 & 10.3 & 3.24 \\
$\left(2 k_{F}, \infty\right)$ & 0.051 & 14.5 & -1.29 & 0.018 & 7.16 & 0.32 \\
$(0, \infty)$ & 1.00 & 41.5 & -9.94 & 1.00 & 46.2 & 15.2 \\
\hline \hline$\left(0, k_{F}\right)$ & 0.861 & 17.7 & 17.7 & 0.912 & 30.4 & 30.4 \\
$\left(k_{F}, 2 k_{F}\right)$ & 0.139 & 6.00 & 6.00 & 0.089 & 5.75 & 5.75 \\
$\left(2 k_{F}, \infty\right)$ & 0.00 & 0.00 & 0.00 & 0.00 & 0.00 & 0.00 \\
$(0, \infty)$ & 1.00 & 23.7 & 23.7 & 1.00 & 36.2 & 36.2 \\
\hline
\end{tabular}

of the distribution around the Fermi surface, $k=k_{F}$, associated to the finite temperature. Notice also that the density $\rho=0.16 \mathrm{fm}^{-3}$ does not correspond to the saturation point of the AV18 potential. Actually, AV18 within SCGF approach at $\mathrm{T}=5 \mathrm{MeV}$, gives a saturation density $\rho=0.19 \mathrm{fm}^{-3}$, smaller than the one obtained in BHF with AV18 (see Table 3). The inclusion of three-body forces should improve the saturation properties (as it happens in the BHF approach) without qualitative changes in the isovector properties 66.

Correlation effects in the momentum distribution are substantially different in SNM than in PNM 68. The effects of the tensor component in the $S-D$ channel in the isospin saturated system induce a large amount of correlations. Consequently, the Fermi surface is quite more depleted for SNM than for PNM (compare the left and right panels in Fig. 44). Characteristic values for these depletions are obtained from the occupation at zero momentum, namely, $n(0) \sim 0.87$ for SNM and $n(0) \sim 0.96$ for PNM. As the momentum distribution is normalized to the total density (see Eq. (26) ), the high-momentum components are also rather different for both systems at the same density. A useful way to characterize these differences is to look at the integrated strength over different regions of momenta:

$$
\phi_{m}\left(k_{i}, k_{f}\right)=\frac{\nu}{2 \pi^{2} \rho} \int_{k_{i}}^{k_{f}} d k k^{m} n(k)
$$

The integral with $m=2$ represents the fractional contribution of a given momentum region to the total density, while the integral with $m=4$ is related to the total kinetic energy of the system.

In Table 11, we report the integrated strengths with $m=2$ for SNM (columns 2 to 4 ) and PNM (columns 5 to 7 ) at $\rho=0.16 \mathrm{fm}^{-3}$ and $T=5$ for the AV18 potential (rows 3 to 6 ) and the FFG (rows 7 to 10). As expected, in SNM there is a substantial depletion of states below the Fermi surface, i.e., only $\sim 75 \%$ of the strength is in 

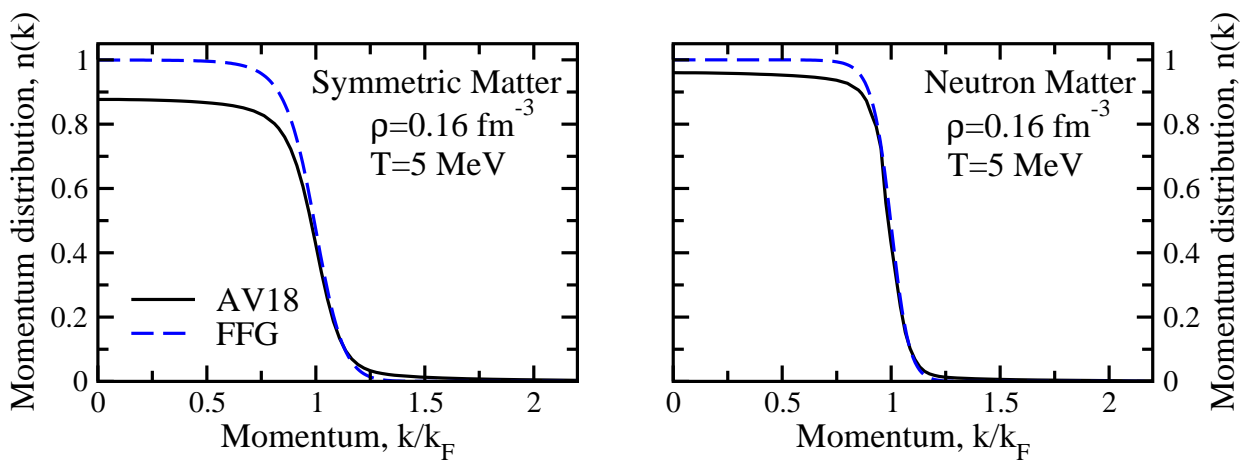

Fig. 4. (Color online) Momentum distribution of SNM (left panel) and PNM (right panel) obtained with the SCGF approximation for AV18 (full lines). The momentum distribution of the FFG (dashed line) obtained in the same conditions are also shown.

the region between 0 and $k_{F}$. Part of the depletion has thermal origin, and the comparison with the FFG in the same momentum region suggests that between $1 / 2$ and $2 / 3$ of the integrated depletion comes from the softening of the Fermi surface due to finite temperature. The effect of correlations is also important in populating states beyond the Fermi surface: for SNM (PNM) there is still a 3-5\% (1-2\%) of strength in the region $k>2 k_{F}$.

The energy per particle is also affected by short-range and tensor correlations. In particular, the kinetic energy,

$$
\frac{\langle K\rangle}{A}=\frac{\nu}{\rho} \int \frac{d^{3} k}{(2 \pi)^{3}} \frac{\hbar^{2} k^{2}}{2 m} n(k),
$$

noticeably increases with respect to the FFG due to the population of high-momentum components. The contributions of the different momentum regions to the kinetic energy of nuclear an neutron matter are also reported in columns 3 and 6 of Table [11. The first thing to notice is that the total integrated values (see rows 4 and 8 of Table 11) of the correlated kinetic energies are larger than those of the FFG. For SNM, dynamical correlations produce and increase of $17 \mathrm{MeV}$ with respect to the FFG, while the increment is only $10 \mathrm{MeV}$ for neutron matter, in agreement with the BHF estimations. Overall, this reinforces the idea that correlations play a smaller role in PNM than in SNM. Paying attention to the different regions, we see that the momentum components beyond $k_{F}$ amount to more than $50 \%$ of the total in SNM, while in PNM they account for around $25 \%$. In contrast, for the FFG at this temperature, the contributions of states above $k_{F}$ is less than $25 \%$ for SNM and less than $15 \%$ for PNM. The FFG strength above the Fermi surface is due to thermal effects, which for low temperatures are mainly localized within a small region around $k_{F}$. As a consequence, there is almost no contribution beyond $2 k_{F}$. Therefore, this contribution in the interacting case can be entirely attributed to NN correlations. In the case of AV18, which is considered to be a hard interaction, the contribution beyond $2 k_{F}$ is even larger than that between the Fermi surface and $2 k_{F}$. Similar analysis have been also performed for other realistic two-body potentials in Ref. 39].
To compute the symmetry energy as the difference of the PNM and SNM energies, we have to resort to the quadratic dependence of the total energy on the isospin asymmetry. This quadratic behavior has been validated in Fig. 2 for the total energy calculated in BHF. The SCGF formalism has been generalized to isospin asymmetric systems and hence we can directly check the quadratic asymmetry dependence of the energy [41]. Moreover, to explore the influence of the high momentum components on the symmetry energy, we have decomposed the symmetry energy in its kinetic and potential energy parts. In the BHF approach, we have used the Hellmann-Feynman theorem to evaluate the kinetic energy. In the SCGF method, the kinetic energy is directly accessible from the momentum distribution and the potential energy can be found from the Galitskii-Migdal-Koltun sum-rule. Consequently, and as a first step before computing isovector properties, we validate the quadratic behavior of both pieces of the total energy with the asymmetry parameter.

In Fig. 5. we show the kinetic, potential and total energy for the AV18 potential at $\rho=0.16 \mathrm{fm}^{-3}$ an $T=5$ $\mathrm{MeV}$ as a function of $\beta^{2}$. In general, the three components seem to have a well-defined parabolic dependence on the asymmetry parameter. The slope of the linear regression reduces to the different components (kinetic, potential and total) of the symmetry energy when the parabolic approximation holds exactly. In the upper panel of Fig. [5 we compare the kinetic energy provided by the AV18 potential and the one of the FFG (triangles). As expected, the correlated kinetic energy is larger than the FFG at all asymmetries. However, the isospin dependence is different than the one of the FFG. While the kinetic energy of AV18 in SNM is $K / A \sim 42 \mathrm{MeV}$ and that of PNM is $\sim 46$ $\mathrm{MeV}$, the FFG gas provides $\sim 24 \mathrm{MeV}$ and $\sim 36 \mathrm{MeV}$ respectively. The difference between the correlated kinetic energies associated to PNM and SNM is smaller than for the FFG. This agrees with the estimate provided by the BHF approach at $T=0$. The small value of the kinetic symmetry energy (clearly smaller than the FFG estimation) is a very noticeable aspect of the decomposition of the symmetry energy in a kinetic and a potential energy component. The origin of this behavior can be related to 


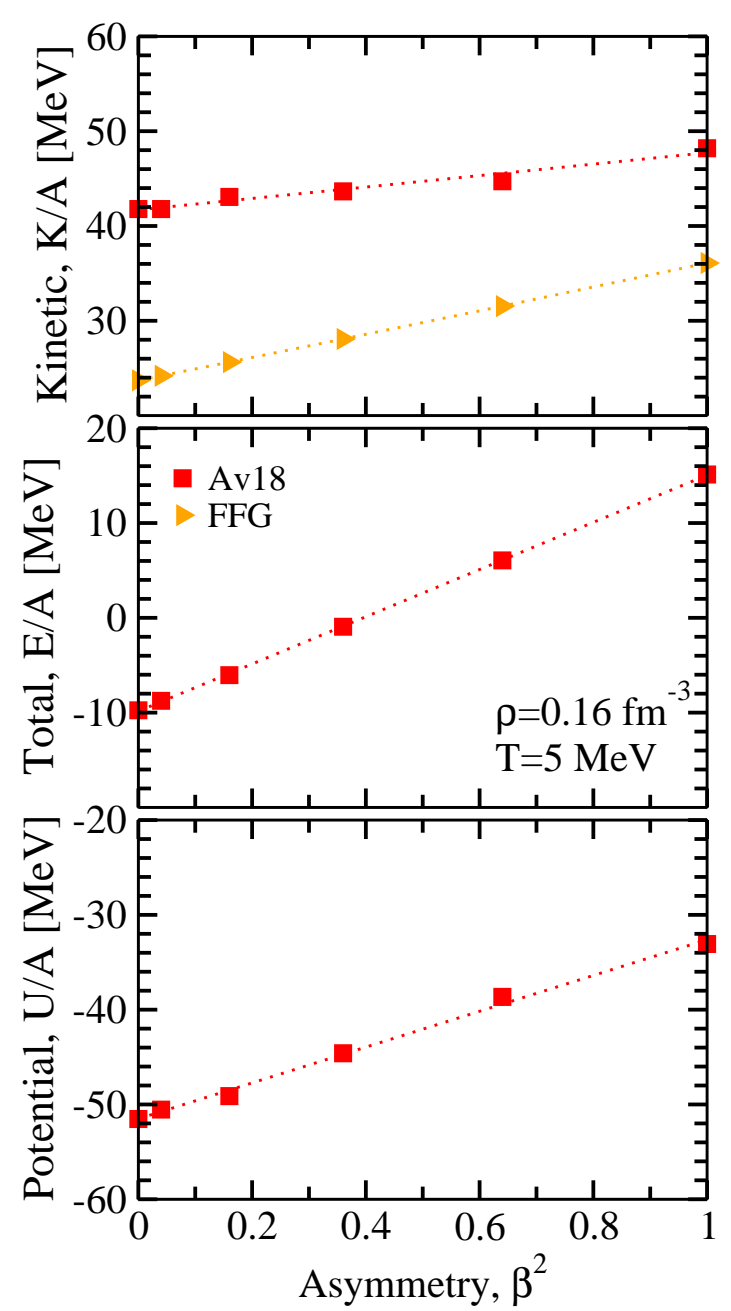

Fig. 5. (Color online) Dependence of the kinetic (upper panel), total (central panel) and potential (lower panel) energy on the asymmetry parameter for the AV18 interaction (squares) at $\rho=0.16 \mathrm{fm}^{-3}$ and $T=5 \mathrm{MeV}$. The triangles in the upper panel give the energy of the FFG in the same conditions. Dotted lines are linear regressions to guide the eye.

the tensor and short range repulsive components of the NN force, that when acting on SNM, induce large correlations and produce an important renormalization of the kinetic energy with respect to the FFG. The absence of this components in PNM (some partial waves are suppressed due to the Pauli principle) reduces the relative enhancement of the kinetic energy. Consequently, the difference in total kinetic energies of PNM and SNM is smaller for the correlated case than for the FFG value.

The asymmetry dependence of the total energy per particle is driven by a balance between the kinetic and potential terms. The size of both contributions is density dependent but, at $\rho=0.16 \mathrm{fm}^{-3}$, the potential term dominates the isospin dependence, as seen in Fig. 6. In other words, the potential energy contribution to the symmetry energy is $20.2 \mathrm{MeV}$, while the kinetic energy part is only $4.9 \mathrm{MeV}$. The total value for the symmetry energy is $S_{2}=25.1 \mathrm{MeV}$, somewhat below the BHF result includ- ing 3BFs. However, one should take into account that the BHF calculation is done at $T=0$ and computed at a saturation density of $0.187 \mathrm{fm}^{-3}$. Regarding thermal effects, the symmetry energy of the FFG can provide an indication of their importance. At $\rho=0.16 \mathrm{fm}^{-3}$, the symmetry energy increases from $12.4 \mathrm{MeV}$ at zero temperature to 13 $\mathrm{MeV}$ at $T=5 \mathrm{MeV}$. Overall, this indicates a very small effect of temperature on the symmetry energy. This is a result of the cancellation of the somewhat similar temperature dependences of symmetric and neutron matter [71].

The total symmetry energy predicted by the SCGF approach is a little below the currently accepted value of $\sim 32 \mathrm{MeV}$ 69. In principle, the inclusion of 3BFs should bring the SCGF results closer to experiment. A first estimation of the effect of $3 \mathrm{BF}$ can be obtained from the BHF calculations including 3BF. Around $\rho=0.16 \mathrm{fm}^{-3}, 3 \mathrm{BF}$ tend to increase the symmetry energy by $3-4 \mathrm{MeV}$. A similar increase in the SCGF case would bring the value closer to experiment.

In general, we see that the symmetry energies provided by SCGF calculations tend to be smaller than the $\mathrm{BHF}$ ones with the same $2 \mathrm{NF}$ force. The origin of this difference can be argued as follows. Compared to the BHF approach, the ladder summation in the SCGF approach includes hole-hole diagrams as well as the full dressing of the intermediate propagators. It is known that both things have an overall repulsive effect in the total energy of the system with respect to the BHF values [70]. This repulsive effect is mostly associated to correlations and, since these are more relevant in SNM, we expect more repulsion in SNM than in PNM. The difference in energies between PNM and SNM is therefore reduced with respect to BHF and the SCGF symmetry energy becomes smaller than the BHF one. Note that, since this repulsive effect increases with density, the slope of the symmetry energy as a function of density is also expected to decrease.

The density dependence of the kinetic and potential energy components of the symmetry energy is shown in Fig. 6. The symmetry energy and its components grow steadily in the density range considered. The potential part is always larger in absolute value than the kinetic one, and dominates the contribution to $S_{2}$. It is interesting to note that the kinetic symmetry energy becomes negative at densities around $0.04-0.08 \mathrm{fm}^{-3}$. One might expect thermal effects to be important in this regime, but the comparison with the FFG once again demonstrates that finite temperature has a negligible effect on the symmetry energy. As a matter of fact, the comparison between the symmetry energy of the FFG at $T=0$ (dashed line) and at $T=5 \mathrm{MeV}$ (solid line) shows that the differences are extremely small (less than $1 \mathrm{MeV}$ ) in the whole density regime. As mentioned above, the small influence of the temperature on the symmetry energy is caused by the relatively similar thermal corrections of SNM and PNM [71. When taking the difference of both energies, one eliminates practically the temperature dependence. Consequently, the negative kinetic symmetry energies at low densities can be considered a NN correlation effect. This 


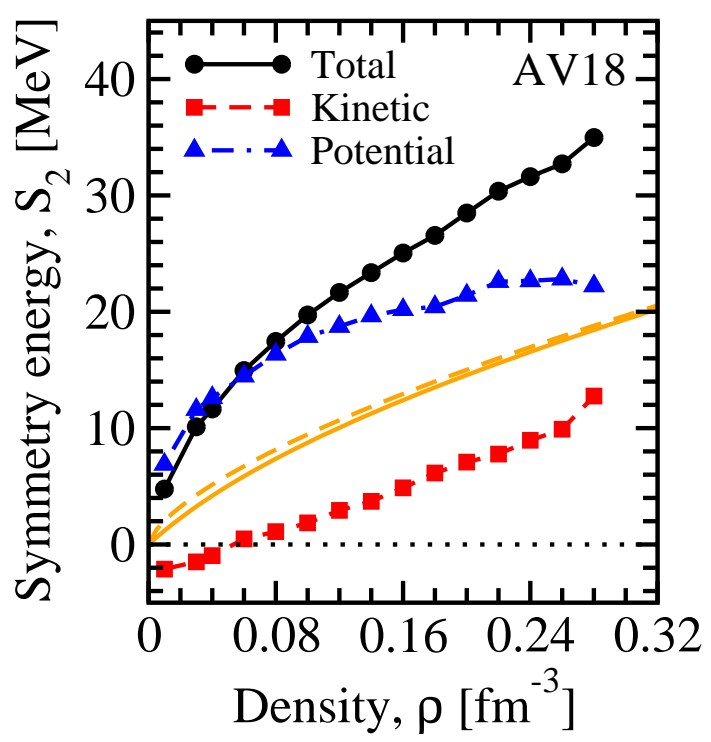

Fig. 6. (Color online) Components of the symmetry energy for the AV18 interaction calculated in the SCGF approach, as a function of density at $T=5 \mathrm{MeV}$. Circles, squares and triangles represent the total, kinetic and potential contributions, respectively. The continuous (dashed) lines correspond to the FFG symmetry energy at $T=5(T=0) \mathrm{MeV}$.

is similar to the BHF case around saturation density and has also been confirmed in other many-body calculations.

\section{Summary and Conclusions}

We have studied the density dependence of the symmetry energy within the microscopic Brueckner-Hartree-Fock and the Self-Consistent Green's Function approaches, using the realistic AV18 NN potential as a starting point. In the BHF case, we have supplemented our calculations with the Urbana IX three-body force. The BHF calculations provide a symmetry energy, $E_{\text {sym }}=34.3 \mathrm{MeV}$, and a value of the slope parameter, $L=66.5 \mathrm{MeV}$, compatible with recent experimental constraints. Using the Hellmann-Feynman theorem, we have evaluated the separate contributions of the different components of the NN interaction to the nuclear symmetry energy and to the slope parameter. This allows for a decomposition of the symmetry energy in a kinetic and potential energy parts. The results show that the potential part gives the main contribution to both $E_{\text {sym }}$ and $L$ and that the kinetic energy contribution is very small. We have also performed a partial-wave as well as a spin-isospin channel decomposition of the potential part of $E_{s y m}$ and $L$, showing that the largest contribution is given by the spin-triplet $(S=1)$ and isospin singlet $(T=0)$ channels. All results point to the dominant role of the tensor component of the NN force, which gives the largest contribution to both $E_{\text {sym }}$ and $L$.

We have completed our analysis by an explicit calculation of the momentum distributions within the SelfConsistent Green's function approach. We have shown how correlations affect differently the momentum distributions of SNM and PNM. We have performed the analysis by quantifying the contribution of momenta beyond the Fermi surface to the kinetic and total energies by using the Galitskii-Migdal-Koltun sum-rule. The change in the high momentum components as the isospin asymmetry is modified confirms the decrease of the kinetic energy component of the symmetry energy with respect to the free Fermi gas. Both changes, namely the decrease in the kinetic energy component of the symmetry energy as well as the change in $n(k)$ with respect to the uncorrelated step function, are used as an indicator of the presence of correlations in SNM and PNM. Also, both measures point towards the same conclusion, namely that correlations play a smaller role in PNM than in SNM. Or, in other words, pure neutron matter is a less correlated system than symmetric nuclear matter.

\section{Acknowledgments}

This work has been supported by the Spanish MICINN Grant No. FIS2011-24154; the Generalitat de Catalunya Grant No. 2009SGR-1289; the Consolider Ingenio 2010 Programme CPAN CSD2007-0042; STFC, through Grants ST/I005528/1 and ST/J000051/1; the R\&DT projects PTDC/FIS/113292/2009 and CERN/FP/123608/2011, developed under the scope of a QREN initiative; UE/FEDER financing, through the COMPETE programme; and by the NEW COMPSTAR, a COST initiative.

\section{References}

1. B. A. Li, L. W. Chen, and C. M. Ko, Phys. Rep. 464, 113 (2008).

2. A. W. Steiner, M. Prakash, J. M. Lattimer, and P.J. Ellis, Phys. Rep. 411, 325 (2005).

3. L. W. Chen, C.M. Ko, and B.A. Li, Phys. Rev. Lett. 94, 032701 (2005).

4. U. Garg et al., Nucl. Phys. A 788, 36 (2007).

5. A. Klimkiewicz et al., Phys. Rev. C 76, 051603(R) (2007);

A. Carbone et al., Phys. Rev. C 81, 041301(R) (2010).

6. P. Danielewicz and J. Lee, Nucl. Phys. A 818, 36 (2009).

7. D. V. Shetty, S. J. Yennello, and G. A. Souliotis, Phys. Rev. C 76, 024606 (2007).

8. B. A. Li, G.-C. Young, and W. Zuo, Phys. Rev. C 71, 014608 (2005).

9. C. Fuchs, Prog. Part. Nucl. Phys. 56, 1 (2006).

10. C. J. Horowitz, S. J. Pollock, P. A. Souder, and R. Michael, Phys. Rev. C 63, 025501 (2001).

11. X. Roca-Maza et al., Phys. Rev. Lett. 106, 252501 (2011).

12. B. A. Brown et al., Phys. Rev. C 76, 034305 (2007).

13. M. Centelles et al., Phys. Rev. Lett. 102, 122502 (2009);

M. Warda et al., Phys. Rev. C 80, 024316 (2010).

14. B. A. Brown, Phys. Rev. Lett. 85, 5296 (2006); S. Typel and B. A. Brown, Phys. Rev. C 64, 027302 (2001)

15. M. B. Tsang, et al., Phys. Rev. C 86, 015803 (2012).

16. H.-J. Schulze, A. Polls, A. Ramos, and I. Vidaña, Phys. Rev. C 73, 058801 (2006). 
17. C. J. Horowitz and J. Piekarewicz, Phys. Rev. Lett. 86, 5647 (2001)

18. J. Xu, L. W. Chen, B. A. Li, and H. R. Ma, Astrophys. J. 697, 1549 (2009); Phys. Rev. C 79, 035802 (2009).

19. Ch. C. Moustakidis, T. Nikšić, G. A. Lalazissis, D. Vretenar, and P. Ring, Phys. Rev. C 81, 065803 (2010).

20. C. Ducoin, J. Margueron, and C. Providência, EPL 91, 32001 (2010); C. Ducoin, J. Margueron, C. Providência, and I. Vidaña, Phys. Rev. C 83, 045810 (2011).

21. J. Lattimer and M. Prakash, Astrophys. J. 550, 426 (2001).

22. J. R. Stone and P. G. Reinhard, Prog. Part. Nucl. Phys. 58, 587 (2007).

23. P. Quentin and H. Flocard, Ann. Rev. Nucl. Part. Sci. 28, 523 (1978).

24. B. D. Serot and J. D. Walecka, Adv. Nucl. Phys. 16, 1 (1986).

25. I. Bombaci and U. Lombardo, Phys. Rev. C 44, 1892 (1991).

26. B. D. Day, Rev. Mod. Phys. 39, 719 (1967); M. Baldo, in M. Baldo (ed.), Nuclear Methods and the Nuclear Equation os State, World Scientific, Singapore, 1999.

27. B. ter Haar and R. Malfliet, Phys. Rep. 149, 207 (1987); Phys. Rev C 36, 1611 (1987); R. Brockmann and R. Machleidt, Phys. Rev. C 42, 1965 (1990).

28. A. Akmal, V. R. Pandharipande, and D. G. Ravenhall, Phys. Rev. C 58, 1804 (1998).

29. A. Fabrocini and S. Fantoni, Phys. Lett. B 298, 263 (1993).

30. W.H. Dickhoff and D.V. Neck, Many-Body Theory Exposed. Propagator description of quantum mechanics in many-body systems, World Scientific, 2005.

31. S. K. Bogner, T. T. S. Kuo, and A. Schwenk, Phys. Rep. 386, 1 (2003).

32. F. Coester, S. Cohen, B. D. Day, and C. M. Vincent, Phys. Rev. C 1, 769 (1970).

33. R. B. Wiringa and S. Pieper, Phys. Rev. Lett. 89, 182501 (2002).

34. R. B. Wiringa, V. G. J. Stoks, and R. Schiavilla, Phys. Rev. C 51, 38 (1995).

35. B. A. Loiseau, Y. Nogami, and C. K. Ross, Nucl. Phys. A 165, 601 (1971); 176, 665(E) (1971); P. Grangé, M. Martzolff, Y. Nogami, D. W. L. Sprung, and C. K. Ross, Phys. Lett. B 60, 237 (1976); M. Baldo and L. Ferreira, Phys. Rev. C 59, 682 (1999).

36. A. Carbone, A. Polls, and A. Rios, arXiv:1307:1889 (2013).

37. I. Vidaña, C. Providência, A. Polls, and A. Rios, Phys. Rev. C 80, 045806 (2009).

38. I. Vidaña, A. Polls, and C. Providência, Phys. Rev. C 84, 062801(R) (2011).

39. A. Carbone, A. Polls, and A. Rios, EPL 97, 22001 (2012).

40. C.-H. Lee, T. T. S. Kuo, G. Q. Li, and G. E. Brown, Phys. Rev. C 57, 3488 (1998).

41. T. Frick, H. Müther, A. Rios, A. Polls, and A. Ramos, Phys. Rev. C 71, 014313 (2005).

42. L. Engvik, M. Hjorth-Jensen, R. Machleidt, H. Müther, and A. Polls, Nucl. Phys. A 627, 85 (1997).

43. V. G. J. Stoks, R. A. M. Klomp, M. C. M. Rentmeester, and J. J. de Swart, Phys. Rev. C 48, 792 (1993).

44. M. Baldo, A. Polls, A. Rios, H.-J. Schulze, and I. Vidaña, Phys. Rev. C 86, 064001 (2012).

45. A. Polls, H. Müther, R. Machleidt, and M. Hjorth-Jensen. Phys. Lett. B 423, 1 (1998).
46. H. Q. Song, M. Baldo, G. Giansiracusa, and U. Lombardo, Phys. Rev. Lett. 81, 1584 (1998).

47. J. P. Jeukenne, A. Lejeunne, and C. Mahaux, Phys. Rep. 25, 83 (1976).

48. X. R. Zhou, G.F. Burgio, U. Lombardo, H.-J. Schulze, and W. Zuo, Phys. Rev. C 69, 018801 (2004).

49. Z. H. Li, U. Lombardo, H.-J. Schulze, and W. Zuo, Phys. Rev. C 77, 034316 (2008).

50. Z. H. Li and H.-J. Schulze, Phys. Rev. C 78, 028801 (2008).

51. X. Viñas, M. Centelles, X. Roca-Maza, and M. Warda, AIP Conf. Proc. 1491, 101 (2012).

52. A. Ekström et al., Phys. Rev. Lett. 110, 192502 (2013).

53. H. Müther and A. Polls, Phys. Rev. C 61, 014304 (1999); R. Sartor, Phys. Rev. C 62, 044318 (2000); R. Sartor, Phys. Rev. C 64, 054303 (2001); I. Bombaci, A, Fabrocini, A. Polls, and I. Vidaña, Phys. Lett. B 609, 232 (2005).

54. H. Hellmann, Einfüring in die Quantenchemie, Leipzig: Franz Deuticke. p. 285 (1937); R. P. Feynman, Phys. Rev. 56, 340 (1939).

55. C. Xu and B. A. Li, arXiv:1104.2075 1 (2011).

56. V. R. Pandharipande and V. K. Garde, Phys. Lett. B 39, 608 (1972)

57. R. B. Wiringa, V. Fiks, and A. Fabrocini, Phys. Rev. C 38, 1010 (1988).

58. G. E. Brown and M. Rho, Phys. Lett. B 237, (1990).

59. H. Müther, M. Prakash, and T. L. Ainsworth, Phys. Lett. B 199, 469 (1987).

60. W. Zuo, I. Bombaci, and U. Lombardo, Phys. Rev. C 60, 024605 (1999); W. Zuo, A. Lejeune, U. Lombardo, and J. F. Mathiot, Eur. Phys. J. A 14, 469 (2002).

61. A. E. L. Dieperink, Y. Dewulf, D. Van Neck, M. Waroquier, and V. Rodin, Phys. Rev. C 68, 064307 (2003).

62. Z. H. Li, U. Lombardo, H.-J. Schulze, W. Zuo, L. W. Chen, and H. R. Ma, Phys. Rev. C 74, 047304 (2006).

63. C. Xu and B. A. Li, Phys. Rev. C 81, 064612 (2010).

64. A. Li and B. A. Li, arXiv:1107.0496v1 (2011).

65. F. Sammarruca, Phys. Rev. C 84, 044307 (2011).

66. V. Somà and P. Bożek, Phys. Rev. C 78, 054003 (2008).

67. T. Alm, G. Röpke, A. Schnell, N. Kwong, and H. Köhler, Phys. Rev. C 53, 2181 (1996).

68. A. Rios, A. Polls, and W. H. Dickhoff, Phys. Rev. C 79, 064308 (2009).

69. M. B. Tsang, W. G. Lynch, and A. W. Steiner, Phys. Rev. Lett. 102, 122701 (2009).

70. H. Müther and A. Polls, Prog. Part. Nucl. Phys. 45, 243 (2000).

71. A. Rios, A. Polls, and I. Vidaña, Phys. Rev. C 79, 025802 (2008). 
